\title{
ORIGINAL ARTICLE \\ Development of a comprehensive survey of sexuality issues including a self-report version of the International Spinal Cord Injury sexual function basic data sets
}

\author{
PW New ${ }^{1,2,3}$ and KE Currie ${ }^{1}$
}

\begin{abstract}
Study design: Questionnaire development, validation and completion.
Objectives: Develop comprehensive survey of sexuality issues including validated self-report versions of the International Spinal Cord Injury male sexual function and female sexual and reproductive function basic data sets (SR-iSCl-sexual function).

Setting: People with spinal cord damage (SCD) living in the community, Australia from August 2013 to June 2014.

Methods: An iterative process involving rehabilitation medicine clinicians, a nurse specialising in sexuality issues in SCD and people with SCD who developed a comprehensive survey that included the SR-iSCl-sexual function. Participants recruitment through spinal rehabilitation review clinic and community organisations that support people with SCD.

Results: Surveys completed by 154 people. Most were male $(n=101,65.6 \%)$. Respondents' median age was 50 years (interquartile range (IQR) 38-58), and they were a median of 10 years (IQR 4-20) after the onset of SCD. Sexual problems unrelated to SCD were reported by $12(8 \%)$ respondents, and $114(n=75.5 \%)$ reported sexual problems because of SCD. Orgasms were much less likely $\left(\chi^{2}=13.1, P=0.006\right)$ to be normal in males $(n=5,5 \%)$ compared with females $(n=11,22 \%)$. Males had significantly worse $\left(\chi^{2}=26.0, P=0.001\right)$ psychogenic genital functioning (normal $\left.n=9,9 \%\right)$ than females (normal $n=13,26 \%$ ) and worse $\left(\chi^{2}=10.8\right.$, $P=0.013)$ reflex genital functioning. Normal ejaculation was reported in only three $(3 \%)$ men. Most $(n=26,52 \%)$ women reported reduced or absent menstruation pattern since SCD.
\end{abstract}

Conclusion: The SR-iSCI-sexual function provides a useful tool for researchers and clinicians to collect information regarding patient-reported sexual functioning after SCD and to facilitate comparative studies.

Spinal Cord (2016) 54, 584-591; doi:10.1038/sc.2015.216; published online 8 December 2015

\section{INTRODUCTION}

Sexual health and sexual functioning are recognised as important domains of health in general and are vital for well-being and quality of life $^{1}$ but often neglected in people with disability. ${ }^{2,3}$ Sexuality changes after spinal cord damage (SCD) from any cause (traumatic or nontraumatic) are common, complex, confounding $\mathrm{g}^{4,5}$ and are a major priority for people with $\mathrm{SCD} .{ }^{6}$ For example, a large survey of people with traumatic spinal cord injury (SCI) from the American National Spinal Cord Injury Database found that sexual functioning was the highest priority for those with paraplegia and the second highest priority (after upper limb function) for people with tetraplegia. ${ }^{\text {? }}$

Studies of sexuality after SCD published to date have focused on people with traumatic SCI. As there is no internationally accepted term for the group of conditions that can cause non-traumatic SCD, ${ }^{8}$ we will use the term spinal cord dysfunction (SCDys) to describe these conditions throughout this article. Although some studies of sexuality have included people with non-traumatic SCDys, ${ }^{9}$ no studies have been located that report separately on the sexual changes in this group. This is important to address because the demographic characteristics and functional outcomes for people with non-traumatic SCDys are different from traumatic SCI, ${ }^{10,11}$ and these differences could potentially impact sexual health outcomes. Furthermore, evidence suggests that in many developed countries the incidence of non-traumatic SCDys is higher than traumatic SCI, ${ }^{12,13}$ and this difference is expected to increase markedly in the coming decades with population ageing. ${ }^{12}$

In recent years, the International Spinal Cord Society (ISCoS) developed the International SCI male sexual function ${ }^{14}$ and female sexual and reproductive function basic data sets. ${ }^{15}$ These are designed to enable a standardised format for collecting and reporting of a minimal amount of information regarding sexual functioning in order to facilitate comparisons between studies, in accordance with the purpose and vision of the International SCI Data Sets project. ${ }^{16}$ The sexual function data sets, however, are worded in a way that is directed at clinicians not patients; hence, they are not appropriate for use as a patient-reported outcome.

The aims of this study were as follows: (1) to develop a self-report version of the International SCI male sexual function and female sexual and reproductive function (SR-iSCI-sexual function) basic data sets, and (2) to include the SR-iSCI-sexual function data sets in a

${ }^{1}$ Spinal Rehabilitation Service, Department of Rehabilitation, Caulfield Hospital, Alfred Health, Melbourne, Victoria, Australia; ${ }^{2}$ Epworth-Monash Rehabilitation Medicine Unit, Southern Medical School, Monash University, Melbourne, Victoria, Australia and ${ }^{3}$ Department of Epidemiology and Preventive Medicine, School of Public Health and Preventive Medicine, Monash University, Melbourne, Victoria, Australia

Correspondence: Dr PW New, Spinal Rehabilitation Service, Department of Rehabilitation, Caulfield Hospital, Alfred Health, 260 Kooyong Road, Caulfield, VIC 3162 , Australia. E-mail: p.new@cgmc.org.au

Received 18 June 2015; revised 27 October 2015; accepted 6 November 2015; published online 8 December 2015 
study of people with SCD residing in the community as part of a comprehensive study of sexuality issues in people with SCD. The objectives were to compare the responses to the SR-iSCI-sexual function data sets from people with traumatic SCI with those who have non-traumatic SCDys and to assess the psychometric properties of the SR-iSCI-sexual function.

\section{MATERIALS AND METHODS}

\section{Study design}

A comprehensive survey was planned that focused on numerous aspects of sexuality in people with SCD living in the community. Principles of good survey design were followed in the design and conduct of this study. ${ }^{17}$

\section{Setting}

The survey commenced on 1 August 2013, and data collection ceased on 30 June 2014. Only people residing in Australia were included.

\section{Participants}

The survey was targeted at adults aged over 18 years of age with SCD from any aetiology - that is both traumatic SCI and non-traumatic SCDys were included. The exclusion criteria were inadequate English language skills to read the consent and survey, multiple sclerosis (because of association with brain damage), congenital aetiology (because the unique circumstances regarding sexual development are very different from those with an acquired SCD), residing in a nursing home or were in a hospital (because these situations adversely impact on establishing and maintaining a sexual relationship and sexual functioning). Potential participants were made aware of these criteria in the survey documentation.

Three methods were used to recruit people with SCD to complete the survey (Figure 1). Patients who had attended the spinal rehabilitation review clinic at the rehabilitation hospital where the authors are based were targeted in two ways. First, those with SCDys who attended the clinic between 1 July 2010 and 30 June $2013(n=136)$ were twice posted (August and September 2013) information about the study, a consent form, a paper version of the survey and a post-paid envelope. Second, from October 2013 a notice about the study was placed in the clinic waiting room (targeting all eligible adults with SCD) that included a URL that directed people to a web-based version of the survey. The web-based version of the survey included information about inclusion and exclusion criteria in the consent section, and this was also repeated on the first data collection page. Third, between October 2013 and June 2014 participants were recruited with the assistance of various Australian State-based organisations that support people with SCD (see Acknowledgements section). These organisations used their websites, newsletters, emails and social media to make readers or members aware of the survey, with the URL for the web-based version of the survey included in these communications. Organisations included information about the study between one and five times in their various communications over the study period.

Non-disabled controls were also recruited to complete questions relevant to them (but not the SR-iSCI-sexual function data sets) for comparison with people with SCD in subsequent publications. These non-disabled controls were recruited by a number of means: participants completing the web-based survey were asked to forward the URL for the study to family and friends; the lead investigator emailed professional and social contacts; and Monash University included information in newsletters emailed to staff and students in the Faculty of Medicine, Nursing and Health Sciences on approximately six occasions over the study period.

\section{Outcome variables included in survey}

Numerous patient-reported outcomes were included in the survey. These were selected on the basis of the clinical expertise of the lead author, a published review of the literature in this area ${ }^{18}$ and our own literature search. The main focus of the project was aspects of sexuality-both spinal cord specific and general disability-related sexuality scales were selected. We included the International SCI core data set, ${ }^{19}$ a self-report classification of non-traumatic SCDys based on the International SCI non-traumatic data sets (available on request $)^{20}$ and the International SCI quality of life basic data set. ${ }^{21}$ Questions were asked about the following areas: gender, including the option for transgender, which was not included in the International SCI core data set; sexual orientation; functional abilities; secondary conditions and complications that are related to $\mathrm{SCD} ;{ }^{22}$ sexual education during rehabilitation; sexual and body esteem, ${ }^{23}$ sexual interest, desire, activities, satisfaction and relationship issues; ${ }^{24-27}$ and sexual abuse. The findings from the above outcomes not

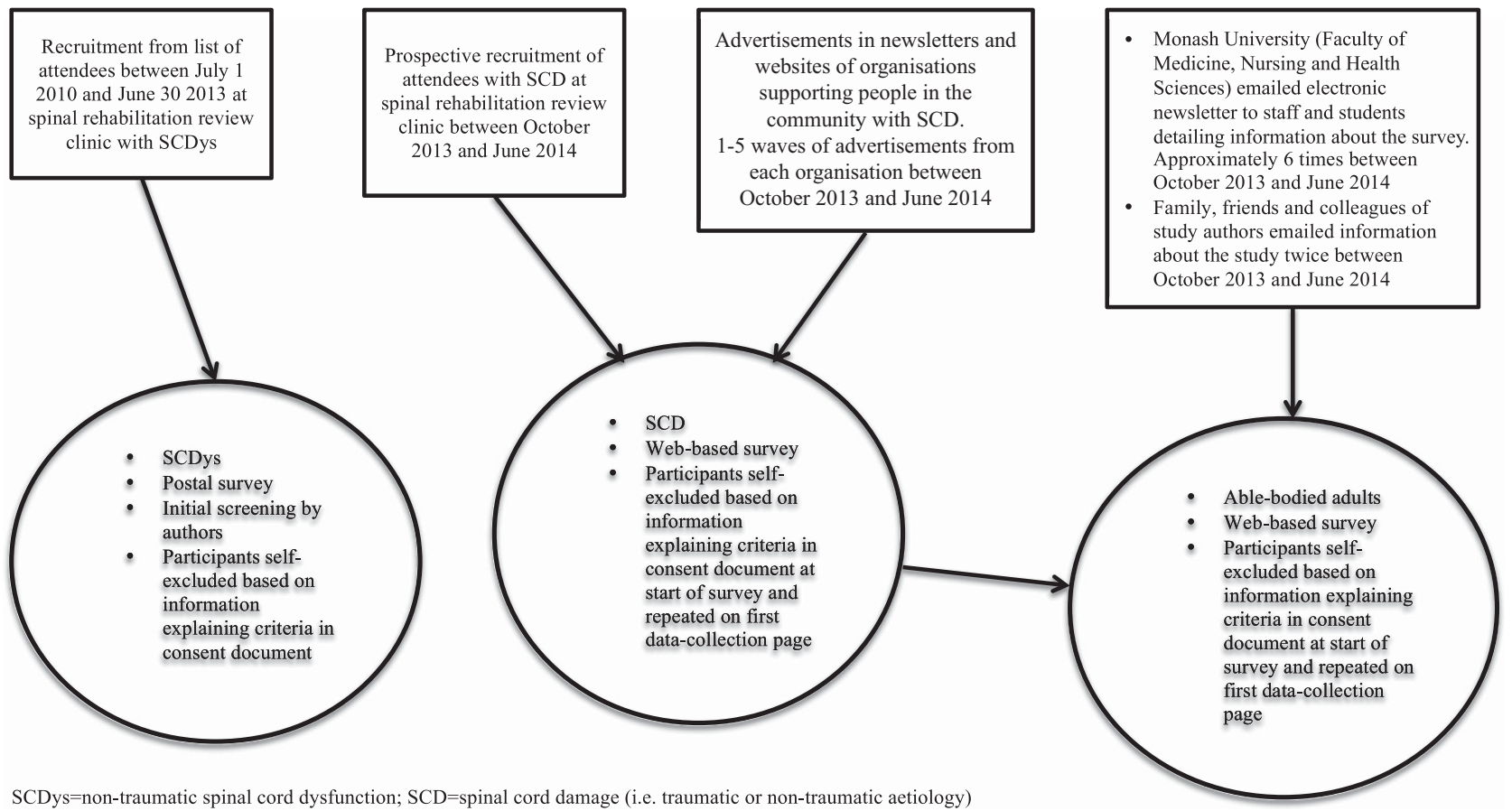

Figure 1 Flowchart illustrating methods of patient recruitment. 
included in the article will be presented in future publications. A copy of the full survey is available from the first author on request.

Some questions in the survey were developed specifically for this project and not based on previously published outcome measures. These included questions

\section{Table 1 Demographic and clinical characteristics}

$\mathrm{n}, \%$

Sexual orientation

Exclusively heterosexual

Gay or lesbian

Bisexual

$146,94.8 \%$

2, $1.3 \%$

$6,3.9 \%$

Current accommodation

Private residence

Assisted living residence (hostel type)

$151,98.0 \%$

Group living situation (for example, community residential unit)

$1,0.7 \%$

$2,1.3 \%$

\section{Current living arrangementa}

Live at home alone

Live at home with partner and/or children

Live at home with family or friend

Live in hostel or supported residential service

$54,35.3 \%$

$62,40.5 \%$

$35,22.9 \%$

2, $1.3 \%$

Highest level of education ${ }^{\text {a }}$
Primary school level only
Secondary school level only
Apprentice, trade certificate or similar
University degree

\section{0}

$54,35.5 \%$

$41,27.0 \%$

$57,37.5 \%$

Employment and income sourcea,b
Employed
Volunteer
Student
Home duties
Unemployed
Age pension
Disability pension
Self-funded retiree
Accident insurance income
Traumatic SCl
Motor vehicle accident (passenger, driver or pedestrian)
Fall
Sport or recreational
Assault (include with weapon)
Other trauma
Non-traumatic SCDys
Disc prolapse or other degenerative causes
Tumour
Ischaemia
Haemorrhage
Infection
Inflammation including transverse myelitis
Other

Other

$60,39.0 \%$

$11,7.1 \%$

$16,10.4 \%$

$10,6.5 \%$

$22,14.3 \%$

$10,6.5 \%$

$54,35.1 \%$

$17,11.0 \%$

3, $1.9 \%$

$115,74.7 \%$

$55,47.8 \%$

$21,18.3 \%$

$26,22.6 \%$

$2,1.7 \%$

$11,9.6 \%$

$39,25.3 \%$

$11,28.2 \%$

$6,15.4 \%$

4, $10.3 \%$

$4,10.3 \%$

$4,10.3 \%$

$5,12.8 \%$

$5,12.8 \%$

Current relationship status ${ }^{a}$

Never married

Married or de facto

Widowed

Separated or divorced

$45,29.6 \%$

$68,44.7 \%$

$5,3.3 \%$

$34,22.4 \%$

Abbreviations: SCDys, spinal cord dysfunction; SCl, spinal cord injury.

a Missing data: current relationship status $n=2$; current living arrangement $n=1$; highest level of

education $n=2$; employment and income source $n=2$.

$\mathrm{b}=$ Total not add up to $100 \%$ as more than one answer possible. that covered functional abilities, sexual education during rehabilitation, sexual abuse and the self-report versions of the International SCI male sexual function ${ }^{14}$ and female sexual and reproductive function data sets. ${ }^{15}$ These novel questions including the rewording of the International SCI sexual function data sets for the SR-iSCI-sexual function data sets were developed in an iterative process involving clinicians experienced in spinal cord rehabilitation (the authors), a nurse specialising in sexuality issues in SCD and six people with SCD living in community. The clinicians did the initial writing of the questions and first rounds of revision. The SR-iSCI-sexual function data sets were reworded to change from a clinician-focused assessment to a more accessible patient-reported outcome using simple to understand explanations for the clinical terms. Feedback was obtained from the nurse and then after further changes from the people with SCD. Further changes were made in response to suggestions from each person before circulating the revised version to the next person with SCD. There were no additional suggestions made from the last two people with SCD regarding the SR-iSCI-sexual function data sets (final version shown in appendix 1).

\section{Sample size}

As this was an exploratory study, there was no predetermined sample size.

\section{Statistical methods}

Continuous variables were not normally distributed and hence were summarised using the median and interquartile range (IQR). The Wilcoxon rank-sum (Mann-Whitney) test was used to calculate differences in the population distribution between continuous variables. Categorical variables were compared using Pearson's chi-square test, with the Fisher exact correction used when small numbers in subgroups indicated that this was appropriate.

It was determined that survey responses would only be included in the analysis if at least one complete sexuality-related outcome measure was completed.

Construct validity of the SR-iSCI-sexual function data sets was assessed using the Spinal Cord Injury Secondary Conditions Scale (SCI-SCS), ${ }^{22}$ which is a valid and reliable scale for self-reporting the impact of secondary conditions following SCD. The scale uses a four-point ordinal rating of how people are affected by various conditions, ranging from 0 (not experienced/insignificant problem) to 3 (significant/chronic problem). The scale includes an item on sexual dysfunction. The sexual function items in the SR-iSCI-sexual function data sets were given scores as follows: $0=$ normal, $1=$ reduced or altered and $2=$ absent. The total score was calculated for men (psychogenic erection, reflex erection, ejaculation and orgasm) and women (psychogenic arousal, reflex arousal and orgasm). Menstruation was not included in the scoring of the SR-iSCI-sexual function data sets for assessment of construct validity as it relates to reproduction not sexual function. The Spearman $\rho$ correlations were calculated between the SCI-SCS item on sexual dysfunction and the total scores from the SR-iSCI-sexual function male and female data sets. The reliability of the SR-iSCI-sexual function data set scores was assessed by testing the internal consistency using Cronbach $\alpha$ with an acceptable level deemed to be 0.70 .

$P$-values of $<0.05$ were deemed statistically significant.

\section{Ethics and software}

All applicable institutional and governmental regulations concerning the ethical use of human volunteers were followed during the course of this research. Stata 12 for Windows (StataCorp, College Station, TX, USA) was used for statistical analysis.

\section{RESULTS}

There were 154 surveys completed by people with SCD included in the analysis. There were 24 completed surveys returned by post (response rate $18 \%$ ). There were 469 surveys initiated via the website, but 123 were excluded ( 70 provided no data at all, 37 failed to complete the specified minimum amount of data and 16 self-reported that they did not meet the inclusion criteria). Non-disabled people completed 220 surveys. 
Table 2 Comparison of demographic and clinical characteristics by cause of spinal cord damage

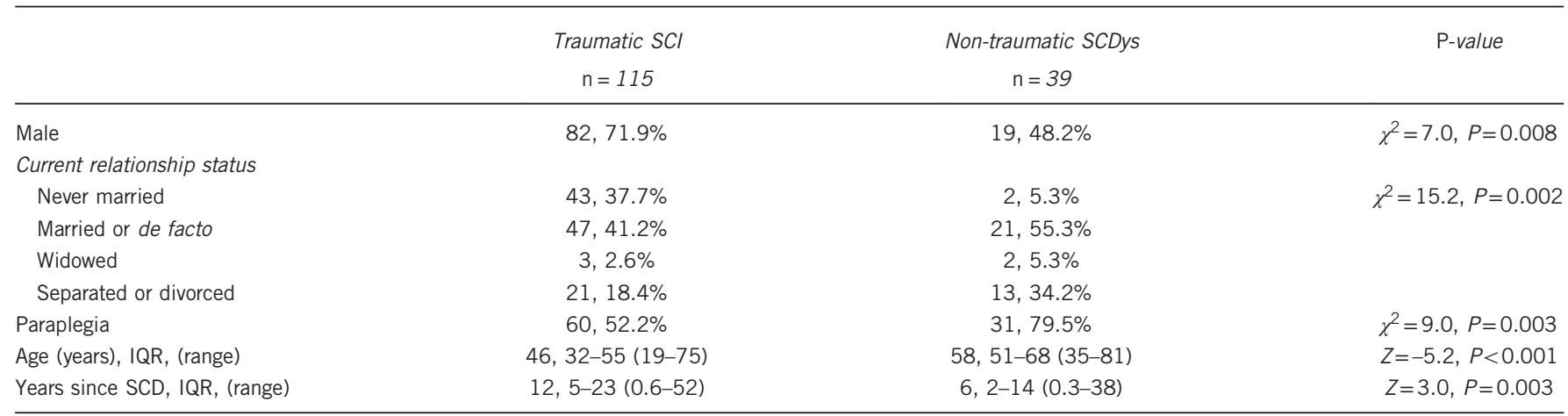

Abbreviations: IQR, interquartile range; SCD,spinal cord damage; SCDys, spinal cord dysfunction; SCI, spinal cord injury.

There were almost twice as many males $(n=101,65.6 \%)$ as females $(n=52,33.7 \%)$ and one participant identified as transgender $(0.7 \%)$. The median age was 50 years (IQR 38-58), and respondents were a median of 10 years (IQR 4-20) following their SCD. Most people $(n=91,59.1 \%)$ had a paraplegic level of injury. The demographic and clinical characteristics of respondents are shown in Table 1. No participants reported their aetiology as 'unknown'. On the basis of the feedback received during the development of the survey from people with SCD, respondents were permitted to indicate more than one response regarding their employment and income sources. Although most respondents $(n=111,72.1 \%)$ indicated only one category of employment or income, two categories were reported by 33 people $(21.4 \%)$, three categories by 6 people $(3.9 \%)$ and four categories were selected by 2 people $(1.3 \%)$.

Differences according to aetiology of SCD

There were significant differences between those with traumatic SCI and non-traumatic SCDys regarding gender, current relationship status, level of SCD, age and number of years since SCD (Table 2).

\section{Self-reports of male sexual function and female sexual and} reproductive function data sets

One hundred fifty-one respondents with SCD completed the SR-iSCI-sexual function data sets. Comparisons between the responses for those with traumatic SCI and non-traumatic SCDys and males and females are shown in Table 3.

Sexual problems or issues unrelated to SCD were reported by 12 $(8 \%)$ of respondents. These problems included the following: erectile dysfunction $(n=4)$, infertility, over-weight, penis size, vaginismus, recent birth of a child, reduced libido and others not specified $(n=3)$.

No differences were found between males and females regarding pre-existing sexual issues unrelated to $\operatorname{SCD}\left(\chi^{2}=1.7, P=0.4\right)$, but sexual dysfunction as a result of SCD was much more likely $\left(\chi^{2}=6.5\right.$, $P=0.04$ ) to be reported by males (sexual dysfunction $n=82,82 \%$; no sexual dysfunction $n=11,11 \%$ unsure $n=7,7 \%$ ) than females (sexual dysfunction $n=32,64 \%$; no sexual dysfunction $n=13,26 \%$ unsure $n=5,10 \%)$. Orgasms were also more likely $\left(\chi^{2}=13.1, P=0.006\right)$ to be abnormal in males (normal $n=5,5 \%$; reduced $n=33,33 \%$; absent $n=52,52 \%$; unknown $n=10,10 \%$ ) than females (normal $n=11$, $22 \%$; reduced $n=20,40 \%$; absent $n=16,32 \%$; unknown $n=3,6 \%$ ). Males had significantly worse psychogenic genital functioning $\left(\chi^{2}=26.0, \quad P=0.001\right)$ and reflex genital functioning $\left(\chi^{2}=10.8\right.$, $P=0.013$ ) than females (Table 3 ).
Psychometric properties of the self-report male and female sexual function data sets

There was a strong correlation between the SCI-SCS sexual dysfunction item and the total scores from the SR-iSCI-sexual function for males $(n=83$, Spearman $\rho=0.40 ; P=0.0002)$ and females $(n=37$, Spearman $\rho=0.41 ; P=0.01)$.

For males, the internal consistency of the SR-iSCI-sexual function was $\alpha=0.73$ (inter-item covariance $=0.16$ ) and for females $\alpha=0.76$ (inter-item covariance $=0.28$ ).

The face validity of the sexual function data sets was confirmed by the process of developing these, as outlined previously. ${ }^{14,15}$ The male SR-iSCI-sexual function score (range 4-12) had a very low floor (1\%) but a mild ceiling $(21 \%)$ effect, whereas the female SR-iSCI-sexual function score (range 3-9) had a low floor (13\%) and a low ceiling $(11 \%)$ effect.

\section{DISCUSSION}

This study has reported on the development of self-report versions of the International SCI male sexual function and female sexual and reproductive function basic data sets. These were used in a sample of people with traumatic and non-traumatic SCD as part of a comprehensive study of sexuality. We found numerous significant differences in the sexual functioning of people with traumatic SCI compared to those with non-traumatic SCDys, as well as genderrelated differences. Our assessment of the psychometric properties of the SR-iSCI-sexual function data sets found no issues of concern.

In this exploratory study there were a number of noteworthy differences in the sexual functioning of people with non-traumatic SCDys and those with traumatic SCI. People with SCDys are more likely to have pre-existing sexual issues and retain the ability to have orgasms and are less likely to be able to ejaculate or menstruate following their SCDys. These differences may be explained by the older age and the greater likelihood of incomplete SCD in people with SCDys.

There are a number of implications of our findings. First, others have suggested that people with SCDys are more likely to have worse sexual adjustment because of the higher likelihood of medical complications that could interfere with a sexual rehabilitation program..$^{28}$ Our results, however, do not support this assertion. If it is accepted that people with SCDys are more likely to have worse sexual adjustment they may be excluded from sexuality rehabilitation programs. We strongly believe that there is no justification for 
Table 3 Results of the self-report versions of the International SCI male sexual function and female sexual and reproductive function basic data sets

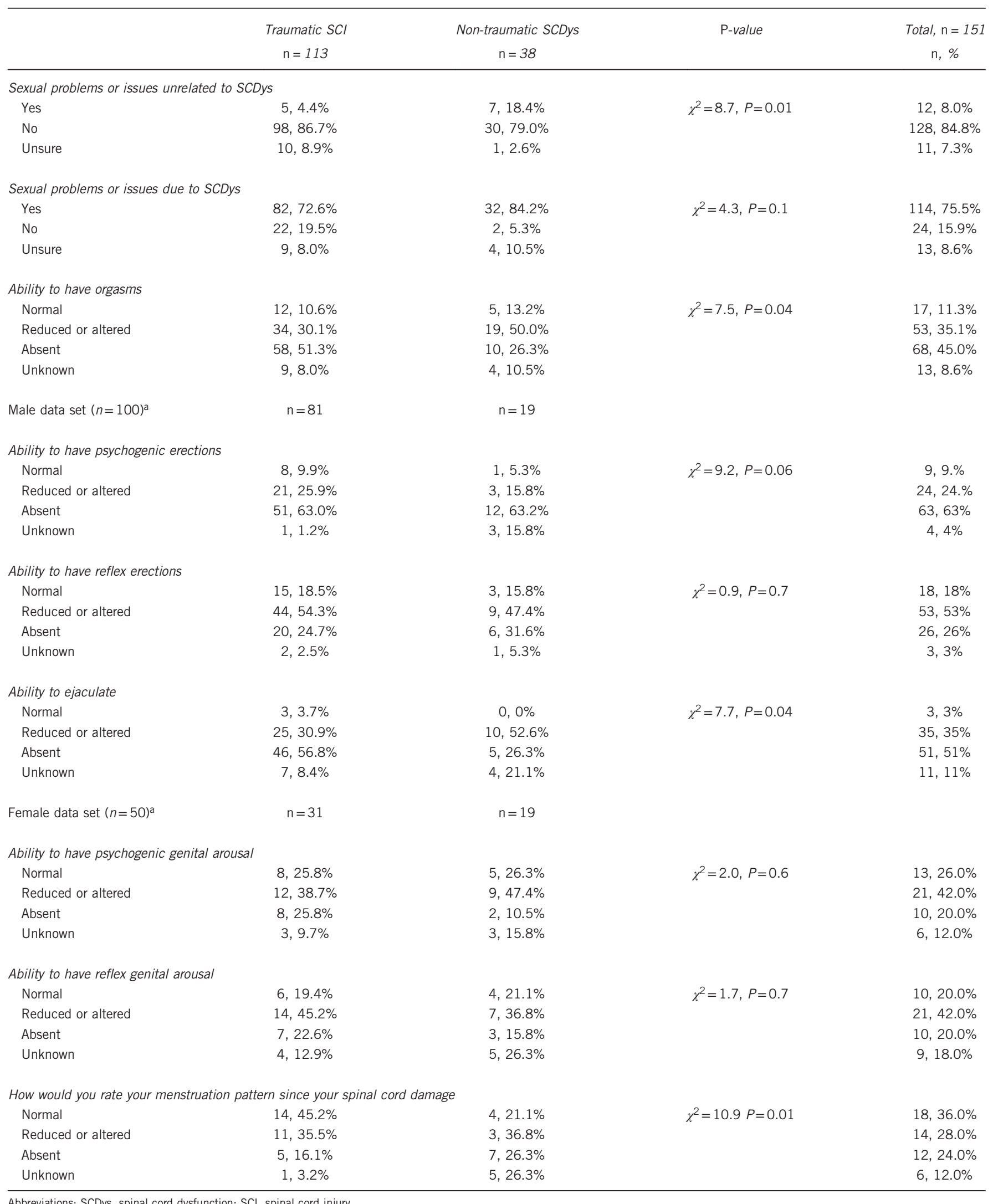

Abbreviations: SCDys, spinal cord dysfunction; SCl, spinal cord injury.

${ }^{\text {aTransgender }}(n=1)$ did not complete either the male or female-specific questions. 
excluding people with SCDys from future studies of sexuality or sexual health following SCD.

Another implication is that our results reinforce the need for sexuality research in people with SCD to include those who are gay, lesbian, bisexual or transgender, although our sample size was too small to report subgroup analyses for these people.

An additional implication of our findings is the justification of the approach taken to allow more than one response to the question regarding employment and income sources. People with SCD engaged in a number of employment and income roles, and these should be assessed with a multiple-response rather than a single-response answer.

Finally, the perspective of people with SCD is recognised as being important in spinal cord research, ${ }^{6}$ and our findings further justify this in relation to sexuality research.

Rehabilitation clinicians working with people who have SCDys need to consider their unique sexuality issues. Careful assessment of sexuality concerns coupled with appropriate education and/or therapeutic intervention should be part of comprehensive SCDys rehabilitation. On the basis of our findings, there are a number of important areas for consideration. Understanding pre-existing medical conditions associated with ageing and how they can affect sexual functioning is important because SCDys is more common in older age and was reported by participants in this study. Given the trend for people with non-traumatic SCDys to be older, ${ }^{10,11}$ they are more likely to have comorbidities that could potentially impact sexual health outcomes. Older men would be less likely to use or tolerate certain erectile dysfunction treatments. Optimising the ejaculatory potential and strategies for dealing with this dysfunction is particularly important for men with SCDys. Awareness of greater problems with menstruation in women with SCDys is needed. Fertility issues are less likely to be a focus of concern in people with SCDys, given the age of this group. Finally, given that involving the partner in assessment and management of sexuality issues is central to satisfaction of the person with SCDys, it is important to be aware of the differences in relationship status of people with SCDys (they are more likely to be married, in a de facto, separated or divorced) compared with those with SCI.

Our findings from the SR-iSCI-sexual function data sets are generally comparable with those from previous studies; however, in all the studies cited below direct comparisons are limited by a wide range in terminology, definitions and wording. This will hopefully be addressed by the uptake among clinicians and researchers of the International SCI data sets. ${ }^{16}$ Erectile dysfunction has been reported by roughly half of $\operatorname{men}^{29,30}$ and ejaculation in less than half. ${ }^{30-32}$ Orgasmic function is typically better preserved in women ${ }^{33,34}$ than in men. ${ }^{30,32}$ Psychogenic arousal ${ }^{33,34}$ and reflex genital arousal ${ }^{34}$ are also typically reduced in women.

The strengths of this study are that it is the first to publish results of the International SCI basic sexual function data sets, and we have developed and validated a self-report version for males and females that can be used by others in future studies. This is also, to our knowledge, the first study to compare in detail the sexual functioning in people with non-traumatic SCDys to those with traumatic SCI.

The epidemiology and the level of injury, both for traumatic SCI and non-traumatic SCDys, are consistent with those from previous reports of SCD in Australia (median age: $\mathrm{SCI}=46, \mathrm{SCDys}=67$; males: $\mathrm{SCI}=72 \%$, SCDys $=53 \%$; paraplegia: $\mathrm{SCI}=50 \%$, SCDys $=69 \%) .{ }^{11}$ The proportions of people identifying as gay, lesbian or bisexual were similar to those in a large population-based study of the Australian population. ${ }^{35}$ On the basis of the above, we believe that it is likely that the results are largely generalisable to people with SCD in Australia, while acknowledging the limitations outlined below. Although we cannot generalise the results here to other countries, we believe that it is possible to use the SR-iSCI-sexual function data sets in other settings.

\section{Limitations and future directions}

Limitations of this study need to be acknowledged, in particular the responder bias to surveys, which is reported to be greater in studies of sexuality. ${ }^{36}$ Nevertheless, alternative study methods are not readily available. A reflection of this responder bias is that the education level reported was higher than that which we would expect to be typical of a population sample of people with SCD, although little is known about education standards in Australians with SCD. The nature of the recruitment methods, which were used for practical purposes, excluded people without computer skills and ready access to a computer, which would bias the sample toward a higher socioeconomic cohort and partially account for the education level. This may also have biased against people with complete tetraplegia and reduced the hand function. However, the use of voice-activated software is readily available to allow people with SCD to use computers independently, and they would have also had the option of completing the survey with the aid of another person if they were comfortable with this. In addition, only those with adequate English language skills would have been able to complete the survey. We estimate that in our unit about $10-15 \%$ of patients admitted would not have adequate English skills to complete the survey. This would create additional bias in the sample. The age and gender mix of respondents, however, suggests that in many aspects our sample is typical of those with SCD. ${ }^{11}$

Additional limitations are that the number of respondents with nontraumatic SCDys was less than anticipated, and the number of females was also relatively low. This reduced the power of the analyses; however, as an exploratory study in this area the analyses serve as a useful benchmark for guiding further research. No American Spinal Injury Association Impairment Scale information was collected from participants because pilot testing indicated that most of the people attending our review clinic did not know this. The response rate from the mailed survey was relatively low, but we were not able to determine to what extent the responder bias influenced the results. There were many people who visited the web-based site without entering any data or the minimum amount of data, but we were not able to determine the reasons for this. No information was collected from women regarding parity or contraceptive use. No information was collected regarding whether participants had used any specific therapies for their sexual dysfunction or what these were. Finally, we cannot be absolutely certain that participants completing the SR-iSCI-sexual function data sets would correctly discriminate whether sexual problems were related or unrelated to their SCD apart from assuming that the unrelated sexuality problems existed before the SCD.

Additional studies are needed to address the dearth of research regarding sexuality issues in people with SCDys. Other directions for future research include using the SR-iSCI-sexual function data sets in other settings and with a larger number of respondents, particularly females and people with SCDys, in order to facilitate comparisons between these subgroups of people with SCD. Further assessment of the psychometric properties of the SR-iSCI-sexual function data sets is needed, in particular the test-retest reliability. Translation into other languages is also appropriate. We advocate for the inclusion of an option for transsexual gender identification in the ISCoS core data set and the addition of sexual orientation in a revised version of the International SCI basic sexual function data sets. 
In conclusion, the SR-iSCI-sexual function data sets provide a useful tool for researchers and clinicians to collect information regarding patient-reported sexual functioning after SCD and to facilitate comparative studies.

\section{DATA ARCHIVING}

There were no data to deposit.

\section{CONFLICT OF INTEREST}

The authors declare no conflict of interest.

\section{ACKNOWLEDGEMENTS}

The following people are thanked for their assistance with the development of the SR-iSCI-sexual function data sets: Lynne Panayiotis, Georgina Fiorentino, Mark Hanson, Mike Short, anonymous, anonymous and Vanessa Hamilton (Sexual Health Nurse Consultant, Austin Health, Melbourne, Australia). Merilyn Seddon (Monash University) and the following organisations are thanked for their assistance with distribution of the survey that included the SR-iSCI-sexual function data sets: AQA (Victoria), Spinal Injuries Association (Queensland), Spinal Cord Injuries Australia (New South Wales), Paraplegic-Quadriplegic Association of WA, ParaQuad NSW, Paraplegic and Quadriplegic Association of South Australia, Spinal Cord Injury Network (Australia). We thank all the participants who completed the survey. Dr Daniel E Rohe, Mayo Clinic, Rochester, MN, USA, is thanked for providing helpful suggestions on an earlier draft of the manuscript. Some of the information in the manuscript was presented at the 53rd ISCoS Annual Scientific Meeting, Maastricht, The Netherlands, on 2 September 2014.

1 World Health Organisation. Defining Sexual Health: Report of a Technical Consultation on Sexual Health 2006.

2 Shakespeare T. Disabled sexuality: toward rights and recognition. Sex Disabil 2000; 18: 159-166.

3 Mall S, Swartz L. Sexuality, disability and human rights: strengthening healthcare for disabled people. S Afr Med J 2012; 102: 792-793.

4 Elliott S. Sexual dysfunction in women with spinal cord injury. In:Lin VW (ed). Spinal Cord Medicine: Principles and Practice. 2nd edn. Demos Medical Publishing: New York, NY 2010; pp 429-437.

5 Elliott S. Sexual dysfunction in men with spinal cord injury. In:Lin VW (ed). Spinal Cord Medicine: Principles and Practice 2nd edn. Demos Medical Publishing: New York, NY 2010; pp 409-428.

6 Simpson L, Eng J, Hsieh J, Wolfe D. Spinal Cord Injury Rehabilitation Evidence (SCIRE) Research Team. The health and life priorities of individuals with spinal cord injury: a systematic review. J Neurotrauma 2012; 29: 1548-1555.

7 Anderson K. Targeting recovery: priorities of the spinal cord-injured population. J Neurotrauma 2004; 21: 1371-1383.

8 New PW, Delafosse V. What to call spinal cord damage not due to trauma? Implications for literature searching. J Spinal Cord Med 2012; 35: 89-95.

9 Kreuter M, Taft C, Siösteen A, Biering-Sørensen F. Women's sexual functioning and sex life after spinal cord injury. Spinal Cord 2011; 49: 154-160.

10 New P, Reeves R, Smith É, Townson A, Eriks-Hoogland I, Gupta A et al. International retrospective comparison of inpatient rehabilitation for patients with spinal cord dysfunction epidemiology and clinical outcomes. Arch Phys Med Rehabil 2015; 96: 1080-1087.

11 New PW, Simmonds F, Stevermuer T. A population-based study comparing traumatic spinal cord injury and non-traumatic spinal cord injury using a National Rehabilitation Database. Spinal Cord 2011; 49: 397-403.

12 New PW, Sundararajan V. Incidence of non-traumatic spinal cord injury in Victoria, Australia: a population-based study and literature review. Spinal Cord 2008; 46: 406-411.

13 Noonan VK, Fingas M, Farry A, Baxter D, Singh A, Fehlings MG et al. The incidence and prevalence of SCl in Canada: a National Perspective. Neuroepidemiology 2012; 38: 219-226.

14 Alexander M, Biering-Sørensen F, Elliott S, Kreuter M, Sønksen J. International spinal cord injury male sexual function basic data set. Spinal Cord 2011; 49: 795-798.

15 Alexander M, Biering-Sørensen F, Elliott S, Kreuter M, Sønksen J. International spinal cord injury female sexual and reproductive function basic data set. Spinal Cord 2011; 49: 787-790.

16 Biering-Sørensen F, Charlifue S, DeVivo M, Noonan V, Post M, Stripling T et al. International spinal cord injury data sets. Spinal Cord 2006; 44: 530-534.

17 Dillman D, Smyth J, Christian L. Internet, Phone, Mail and Mixed-Mode Surveys: The Tailored Design Method. 4 ed. John Wiley \& Sons Inc.: Hoboken, NJ 2014.
18 Abramson C, McBride K, Konnyu K, Elliott S. Sexual health outcome measures for individuals with a spinal cord injury: a systematic review. Spinal Cord 2008; 46: 320-324.

19 DeVivo M, Biering-Sørensen F, Charlifue S, Noonan V, Post M, Stripling T et al. International spinal cord injury core data set. Spinal Cord 2006; 44: 535-540.

20 New PW, Marshall R. International spinal cord injury data sets for non-traumatic spinal cord injury. Spinal Cord 2014; 52: 123-132.

21 Charlifue S, Post MW, Biering-Sørensen F, Catz A, Dijkers M, Geyh S et al. International spinal cord injury quality of life Basic Data Set. Spinal Cord 2012; 50: 672-675.

22 Kalpakjian C, Scelza W, Forchheimer M, Toussaint L. Preliminary reliability and validity of a spinal cord injury secondary conditions scale. J Spinal Cord Med 2007; 30: 131-139.

23 Taleporos G, McCabe MP. Development and validation of the physical disability sexual and body esteem scale. Sex Disabil 2002; 20: 159-176.

24 Kreuter M, Sullivan M, Siösteen A. Sexual adjustment after spinal cord injury (SCl) focusing on partner experiences. Paraplegia 1994; 32: 225-235.

25 Kreuter M, Sullivan M, Siösteen A. Sexual adjustment after spinal cord injurycomparison of partner experiences in pre- and postinjury relationships. Paraplegia 1994: 32: 759-770.

26 Kreuter M, Sullivan M, Siösteen A. Sexual adjustment and quality of relationships in spinal paraplegia: a controlled study. Arch Phys Med Rehabil 1996; 77: 541-548.

27 Siösteen A, Lundqvist C, Blomstrand C, Sullivan L, Sullivan IM. Sexual ability, activity, attitudes and satisfaction as part of adjustment in spinal cord-injured subjects. Paraplegia 1990; 28: 285-295.

28 Lombardi G, Del Popolo G, Macchiarella A, Mencarini M, Celso M. Sexual rehabilitation in women with spinal cord injury: a critical review of the literature. Spinal Cord 2010; 48: 842-29.

29 Biering-Sørensen F, Sønksen J. Penile erection in men with spinal cord or cauda equina lesions. Semin Neurol 1992; 12: 98-105.

30 Anderson K, Borisoff J, Johnson R, Stiens S, Elliott S. Long-term effects of spinal cord injury on sexual function in men: implications for neuroplasticity. Spinal Cord 2007; 45: 338-348.

31 Biering-Sørensen F, Sønksen J. Fertility in men with spinal cord or cauda equina lesions. Semin Neurol 1992; 12: 106-114.

32 Alexander C, Sipski M, Findley T. Sexual activities, desire, and satisfaction in males pre- and post-spinal cord injury. Arch Sex Behav 1993; 22: 217-228.

33 Sipski M, Alexander C. Sexual activities, response, and satisfaction in women pre- and post-spinal cord injury. Arch Phys Med Rehabil 1993; 74: 1025-1029.

34 Anderson K, Borisoff J, Johnson R, Stiens S, Elliott S. Spinal cord injury influences psychogenic as well as physical components of female sexual ability. Spinal Cord 2007; 45: 349-359.

35 Grulich AE, de Visser RO, Badcock PB, Smith AMA, Heywood W, Richters J et al. Homosexual experience and recent homosexual encounters: the Second Australian Study of Health and Relationships. Sexual Health 2014; 11: 439-450.

36 Strassberg DS, Lowe K. Volunteer bias in sexuality research. Arch Sex Behav 1995; 24: 369-382.

\section{APPENDIX 1}

Self-report versions of the International Spinal Cord Society male sexual function and female sexual and reproductive function data sets

\section{QUESTIONS IN BOTH MALE AND FEMALE DATA SETS}

Do you have any sexual problems or issues unrelated to your spinal cord damage?

_ Yes, please specify

- No

_ Unsure

Do you have any sexual problems or issues due to your spinal cord damage?

- Yes

- No

_ Unsure

How would you rate your ability to have an orgasm?

Normal

- Reduced/altered

- Absent

Unknown

\section{MALES DATA SET}

How would you rate your ability to have psychogenic erections? (A psychogenic erection is an erection that occurs without any physical touching. This may occur if a man thinks of something sexual or sometimes it just happens on its own)? 
— Normal

_ Reduced/altered

_ Absent

— Unknown

How would you rate your ability to have reflex erections (This is an erection that occurs because of physical stimulation or touch)?

_ Normal

— Reduced/altered

Absent

_ Unknown

How would you rate your ability to ejaculate?

Normal

Reduced/altered

_ Absent

_ Unknown

\section{FEMALES DATA SET}

How would you rate your ability to have psychogenic genital arousal (This is when genital changes and lubrication occur without any physical touching. This may occur if a woman thinks of something sexual or sometimes it just happens on its own)?

$$
\begin{aligned}
& \text { Normal } \\
& \text { — Reduced/altered } \\
& \text { — Absent } \\
& \text { _ Unknown }
\end{aligned}
$$

How would you rate your ability to have reflex genital arousal (This is when genital changes and lubrication occur because of physical stimulation or touch)?

_ Normal

— Reduced/altered

Absent

_ Unknown

How would you rate your current menstruation pattern?

Normal

_ Reduced/altered

Absent

_ Unknown 